\title{
LETTER
}

\section{Improvement in functional abilities at ICU discharge is feasible without prolongation of the length of stay ICU}

\author{
Matty Koopmans* ${ }^{*}$, Luuk Vermei, Anja van Wieren, Nynke Bruins, Corine M. de Jager and E. Christiaan Boerma
}

@ 2016 The Author(s). This article is published with open access at Springerlink.com

\section{Dear Editor,}

Although survival of patients treated in the intensive care unit (ICU) is increasing, a prolonged ICU stay is still associated with severe physical and mental problems. Disabilities associated with a long ICU stay often result in readmissions, reduction in quality of life and substantial use of healthcare resources [1,2]. Although specific therapies to prevent this form of unfavourable outcome seem to be lacking, substantial progress is made in the reduction of functional loss, associated with standard ICU treatment. In 2013 we adopted a set of previously described interventions aiming to prevent post-ICU disabilities. This included reduction of sedation, enabling speech during mechanical ventilation, early mobilisation of ventilator-dependent patients and non-drug treatment of delirium [3, 4]. The aim of the present study is to investigate whether the introduction of this set of interventions is associated with an increase in functional abilities after ICU discharge. And, if so, what will be the impact on the length of stay (LOS) in the ICU.

In order to assess the severity of post-ICU disabilities we systematically assessed the Barthel index (BI) in all patients $24-48 \mathrm{~h}$ post ICU discharge [5]. This BI is an instrument to semi-quantitatively score patients' abilities on 10 domains of daily life activities, such as feeding, mobility, incontinence, transfer, etc. We performed a single-centre retrospective cohort study, including all patients admitted to the ICU between 2012 and 2014 and a LOS of greater than $48 \mathrm{~h}$. Patients readmitted to the ICU were excluded, but their data remained part of the analysis with respect to the first ICU admission. Patients' baseline characteristics were recorded and descriptive statistics as well as uni- and multivariate analyses were applied. In this 3-year period we included 825 patients. At baseline, patients were not different in age and gender. Severity of illness expressed as APACHE III score did not differ over time from 69 [54-87] in 2012 to 73 [57-95] in 2014, $p=0.13$. In this period the BI increased from 9 [4-14] in 2012 to 11 [6-16] in 2014, $p<0.001$ (Fig. 1). The observed increase in BI between 2012 and 2014 was also significant in all individual domains of the BI. After correction for baseline confounders in a multivariate analysis, the BI in the cohort year 2012 remained significantly lower in comparison to 2013 and 2014. In addition, the duration of mechanical ventilation decreased significantly between 2012 and 2014 from 1.8 [0.5-5.5] to $1.6[0.1-3.8]$ days, $p=0.005$. However, the observed improvement in functional ability over time was not associated with a rise in ICU LOS (7 [5-12] versus 6.5 [4-13] respectively, $p=0.56$ ).

In conclusion, our data suggest that the introduction of a set of interventions aimed at the reduction in ICU-acquired disabilities is indeed associated with an improvement in independence during daily life activities.

*Correspondence: matty.koopmans@znb.nl 


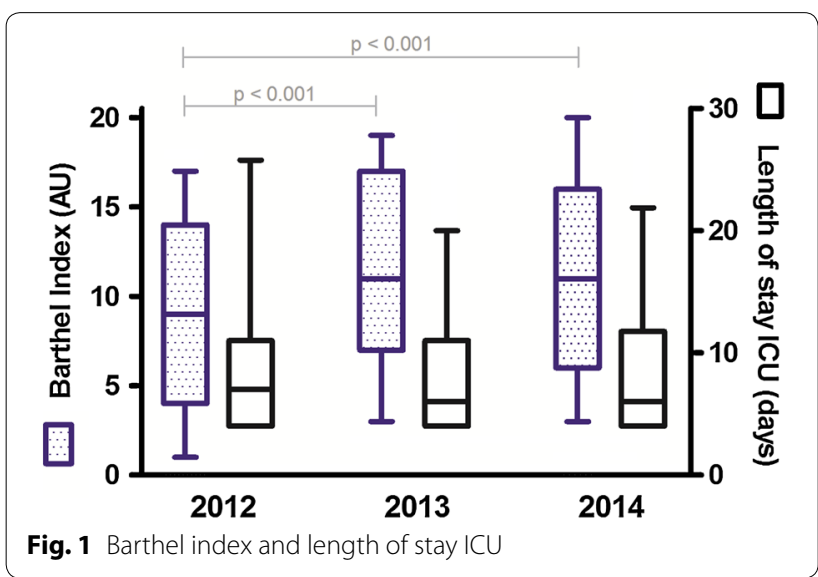

Moreover, this improvement of functional abilities does not come at the price of an extended length of stay in the ICU.

\section{Compliance with ethical standards}

\section{Conflicts of interest}

The authors declare that they have no conflict of interest.

\section{Ethics}

As a result of the non-interventional character of the study, the institutional review board (RTPO, Medical Centre Leeuwarden) approved this study, without the need for informed consent (nWMO 2015-143).
Open Access This article is distributed under the terms of the Creative Commons Attribution-NonCommercial 4.0 International License (http:// creativecommons.org/licenses/by-nc/4.0/), which permits any noncommercial use, distribution, and reproduction in any medium, provided you give appropriate credit to the original author(s) and the source, provide a link to the Creative Commons license, and indicate if changes were made.

Accepted: 20 September 2016

Published online: 29 September 2016

References

1. Kaukonen KM, Bailey M, Suzuki S, Pilcher D, Bellomo R (2014) Mortality related to severe sepsis and septic shock among critically ill patients in Australia and New Zealand, 2000-2012. JAMA 311:1308-1316. doi:10.1001/jama.2014.263

2. Douglas SL, Daly BJ, Brennan PF, Gordon NH, Uthis P (2001) Hospital readmission among long-term ventilator patients. Chest 120:1278-1286

3. Egbers PH, Bultsma R, Middelkamp H, Boerma EC (2014) Enabling speech in ICU patients during mechanical ventilation. Intensive Care Med 40:1057-1058. doi:10.1007/s00134-014-3315-7

4. Svenningsen $H$ (2013) Associations between sedation, delirium and posttraumatic stress disorder and their impact on quality of life and memories following discharge from an intensive care unit. Dan Med J 60:B4630

5. Collin C, Wade DT, Davies S, Horne V (1988) The Barthel ADL index: a reliability study. Int Disabil Stud 10:61-63 